\title{
ANTICIPATORY GRIP FORCE CONTROL USING A CEREBELLAR MODEL
}

\author{
J. R. DE GRUIJL, ${ }^{\text {a*}}$ P. VAN DER SMAGT ${ }^{\mathrm{b}}$ \\ AND C. I. DE ZEEUW ${ }^{\mathrm{a}, \mathrm{c}}$ \\ ${ }^{a}$ Netherlands Institute for Neuroscience, Royal Netherlands Academy \\ of Arts and Sciences, Meibergdreef 47, 1105 BA Amsterdam, The \\ Netherlands \\ ${ }^{b}$ Deutsches Zentrum für Luft- und Raumfahrt (DLR)/Institute of Robot- \\ ics and Mechatronics, PO Box 1116, 82230 Wessling, Germany \\ ${ }^{c}$ Department of Neuroscience, Erasmus MC, 3000 DR Rotterdam, The \\ Netherlands
}

\begin{abstract}
Grip force modulation has a rich history of research, but the results remain to be integrated as a neurocomputational model and applied in a robotic system. Adaptive grip force control as exhibited by humans would enable robots to handle objects with sufficient yet minimal force, thus minimizing the risk of crushing objects or inadvertently dropping them. We investigated the feasibility of grip force control by means of a biological neural approach to ascertain the possibilities for future application in robotics. As the cerebellum appears crucial for adequate grip force control, we tested a computational model of the olivo-cerebellar system. This model takes into account that the processing of sensory signals introduces a $100 \mathrm{~ms}$ delay, and because of this delay, the system needs to learn anticipatory rather than feedback control. For training, we considered three scenarios for feedback information: (1) grip force error estimation, (2) sensory input on deformation of the fingertips, and (3) as a control, noise. The system was trained on a data set consisting of force and acceleration recordings from human test subjects. Our results show that the cerebellar model is capable of learning and performing anticipatory grip force control closely resembling that of human test subjects despite the delay. The system performs best if the delayed feedback signal carries an error estimation, but it can also perform well when sensory data are used instead. Thus, these tests indicate that a cerebellar neural network can indeed serve well in anticipatory grip force control not only in a biological but also in an artificial system. (c) 2009 Published by Elsevier Ltd on behalf of IBRO.
\end{abstract}

Key words: cerebellum, grip force, motor learning, computational model.

In our daily lives, we frequently handle objects without much thought. However, applying an adequate amount of grip force to an object being handled requires tight coordination with the dynamics of other applied forces. Let us consider the case of a robot handling an object. Its task is to simply hold it in its hand while the arm or body is being

*Corresponding author. Tel: +31-0-205668404.

E-mail address: j.de.gruijl@nin.knaw.nl (J. R. de Gruijl).

Abbreviations: BC, basket cell; CF, climbing fiber; DCN, deep cerebellar nuclei; GC, granule cell; GO, Golgi cell; IO, inferior olivary nucleus; LTD, long-term depression; LTP, long-term potentiation; MF, mossy fiber; MSE, mean squared error; PC, Purkinje cell; PF, parallel fiber; SC, stellate cell.

0306-4522/09 @ 2009 Published by Elsevier Ltd on behalf of IBRO. doi:10.1016/j.neuroscience.2009.02.041 moved. The robot would ideally perform this task with minimal grip force, thus preserving energy and-more importantly-minimizing the risk of crushing the object, which is a realistic risk when, e.g. holding a Styrofoam cup. Unfortunately, using the same low amount of force constantly will not suffice, because any movement of the robot hand or arm will automatically apply a force to any object that is being handled. Because of this, inertial loads can fluctuate, possibly causing the object to slip from the robot's grasp and fall. Thus, grip force needs to be modified on occasion. Relying solely on sensory feedback to solve this problem may not be a feasible approach, since the processing of sensory data may well take too long, especially if the movements are fast. In short, the system has to anticipate changes in load force based on its movements and adjust its grip accordingly, so as to prevent to crush or drop the object it is holding.

Grip force modulation has been studied extensively in humans. Healthy human test subjects tend to hold objects with near-minimal grip force (Johansson and Westling, 1984) and adjust this force in synchrony with or even prior to the object's load force changes during movement, indicating anticipation (Flanagan and Wing, 1993; Flanagan et al., 2003). Indeed, anticipation is needed since sensory feedback arrives with a delay. This delay is also reflected by the fact that changes in motor behavior due to an unexpected event usually occur at a latency of about 100 ms (Johansson and Westling, 1987; Cole and Abbs, 1988).

Positron emission tomography (PET) scans reveal that the cerebellum plays a major role during grip force-load force coupled tasks (Boecker et al., 2005). Patients with a damaged cerebellum lack the tight coordination of grip and load force, often exerting more grip force than needed and having difficulties in timing motor actions e.g. to compensate for predictable perturbations (Babin-Ratté et al., 1999; Nowak et al., 2002; Serrien and Wiesendanger, 1999). Lesions in other areas involved in motor control such as the cerebral cortex or striatum result in paralysis or involuntary movement rather than a loss of coordination.

The cerebellum has a well-known neural structure and plays an important role in motor control in general (De Zeeuw and Yeo, 2005). Thus, it is not surprising that its network served as the basis for numerous computational models (Albus, 1975; Medina et al., 2000; Spoelstra et al., 2000; Porrill et al., 2004; Yamazaki and Tanaka, 2007). Here, we investigated the feasibility of using a cerebellar model to control grip force.

\section{Outline}

In the following sections, a brief overview of the cerebellum and interpretation of its functionality will be given first. After 
that, the dataset and model used for the current work will be described. Finally, the results are presented and the current work will be discussed.

\section{The olivo-cerebellar system}

The cerebellum has two main input channels: the mossy fibers (MF) and the climbing fibers (CF). Both inputs are excitatory. The first carry signals originating from many different regions such as the pons, lower brainstem regions, and spinal cord, while the second solely carries signals from the inferior olivary nucleus $(I O)$ in the ventral medulla oblongata. Interestingly, the inferior olive receives its inputs directly or indirectly from many of the regions that also give rise to one of the MF projections (De Zeeuw et al., 1998). Unlike the IO signals, which relay all or none signals at the Purkinje cell (PC) level, the MF signals undergo some form of recoding before arriving in the PC layer: they terminate in the granule cell (GC) layer where combinations of various MF signals are integrated with a feedback from the Golgi cells (GO) into parallel fiber (PF) signals, which in turn are carried to the PCs. Because the number of PFs is much higher than that of the MFs, it has been suggested that expansion recoding occurs (Albus, 1975; Spoelstra et al., 2000). The PFs and CFs co-terminate in the PC layer of the cerebellum, where synaptic weights are modified. It is generally believed that for this single layer of cells, the PF signals provide a current motor command and delayed sensory context, whereas the CFs may carry an error signal. This take on the cerebellum is known as the Marr-Albus-Ito hypothesis (Marr, 1969; Albus, 1971; Ito, 1984). The output of the PCs is sent to the deep cerebellar nuclei (DCN), where they merge with the input from both MF and CF collaterals and where the final output of the cerebellum is generated. Thus, the cerebellum is reminiscent of a simple perceptron and as such it may learn to apply an inverse or forward model based on its input. Inverse models enable a system to generate the desired output by producing the command that achieves a given desired state based on the current state of the system. Forward models can overcome feedback delays by predicting the result of a current command, given the current state of the system. Both models would have to be under continuous revision based on sensory input. There are indications that both types of model are used by the motor apparatus, and it has been suggested that the cerebellum may function as an inverse model that overcomes time delays using forward models (Wolpert et al., 1998).

The complexity of its cellular configurations and connections, among other things, sets the cerebellum apart from a standard perceptron. For instance, the GCs are all excitatory, while all PCs generate inhibitory output. In addition, both the granular layer and molecular layer of the cerebellar cortex have inhibitory interneurons; these include the GOs, which inhibit the GCs, and the stellate cells (SC) and basket cells (BC), which inhibit the PCs.

The interaction of the cerebellum with the $I O$ also sets it apart from a simple perceptron. PCs inhibit both excitatory and inhibitory neurons in the DCN, the latter ones of which inhibit the 10 neurons that provide the CFs to the
PCs. Thus, the result is a topographically organized loop that is specific down to the cellular level. Interestingly, the olivary neurons are electrotonically coupled to each other and have a tendency to oscillate (Van Der Giessen et al., 2008). Moreover, due to their conductances, olivary neurons have a very low firing frequency with a maximum rate of $10 \mathrm{~Hz}$ (Llinás and Volkind, 1973) and a spontaneous activity of $2 \mathrm{~Hz}$ or less (Yeo and Hesslow, 1998). This arrangement implies that the cerebellum receives relatively few corrective signals, even when learning a new task.

\section{The computational model}

Input data. To provide the system with input and test the system's output, we constructed our own dataset of human grip force behavior with recordings acquired from nine healthy adult test subjects $(A, B, C, D, E, F, G, H$ and I). During right-handed point-to-point vertical arm movements with an amplitude of approximately $30 \mathrm{~cm}$, pausing after every upward and downward motion (as described in Nowak et al., 2002), participants held a manipulandum (see Fig. 1) that registered accelerations in 3D, grip force and torque on the grip force axis. The manipulandum could be outfitted with surfaces of varying roughness to establish different baseline grip force levels. These materials included, in order of increasing roughness, paper, Plexiglas and sandpaper. A total of 35 traces, each consisting of five downward and five upward movements with pauses in between, spanning approximately $20 \mathrm{~s}$ on average, was gathered and used. Appendix A contains an overview of the number of traces per subject and surface texture, as

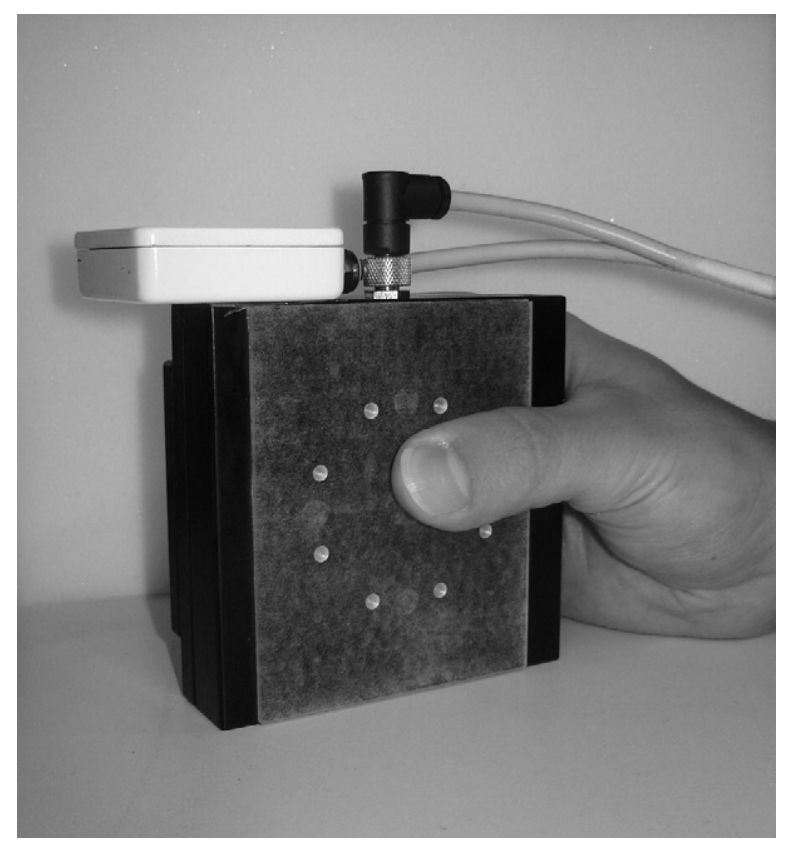

Fig. 1. The manipulandum with Plexiglas surfaces being held. The black sensor registers force and torque exerted on the gripped surfaces. The top-mounted white sensor registers accelerations in three dimensional space. 
well as statistics describing grip force differences between surface textures.

Olivo-cerebellar model. Even though the cerebellum seems similar in structure to a perceptron, we did not use one for the current model. Besides the fact that selecting a biologically plausible learning rule is highly problematic, our preliminary results showed that the task of grip force control cannot be learned by a conventional perceptron, even when it is supplied with a delayed feedback signal. This is caused in part by the fact that its computational elements generate their output based solely on their current input and synaptic weights. Since the input can be rather noisy, the output can be noisy. Because the motor output is fed back into the system with a 100 ms delay, the system's output remains noisy. Some representation of past activations could ameliorate this problem.

It has been suggested that the cerebellum functions like a liquid state machine (Yamazaki and Tanaka, 2007). A liquid state machine consists of a feedforward learning layer incapable of memory representation that reads its input patterns from the activations in a recurrent network capable of retaining information of past events (Maass et al., 2002). In this case, the GCs and GOs would form the recurrent network and the $\mathrm{PC}$ layer the feedforward readout network. However, the notion of the GC layer retaining information by means of recurrent connections seems unlikely, due to the inhibitory nature of the GC/GO recurrent connection.

We used a modified version of a cerebellar model by Spoelstra et al. (2000), which was originally used for correcting the motor output of a simulated robot arm during point-to-point movements by learning an inverse model. Cells are modeled as leaky integrators, causing an intrinsic retention of information (or smoothing of activity) in the form of a decaying membrane potential

$$
\begin{gathered}
\tau \frac{d m}{d t}=-m+x \\
F(m)=F_{\text {max }} \frac{1}{1+e^{-s(m-o)}}
\end{gathered}
$$

where $m$ denotes the membrane potential, $x$ the synaptic input, $F$ the firing rate and $s$ and $o$ the slope and the offset of the specific neuron type's firing properties. Values for these parameters are given in Table 1. In Fig. 2 a graphical overview of the system's global architecture is supplied.

Only the olivary neurons are not simulated as leaky integrators; these are represented as leaky integrate-and-

Table 1. Overview of the parameter values used to model different cell types as leaky integrators

\begin{tabular}{lcclcc}
\hline & GC & GO & PC & DCN & s.c./BC \\
\hline Time constant $\tau$ & 0.02 & 0.05 & 0.02 & 0.02 & 0.05 \\
Slope $s$ & 8 & 0.5 & 0.005 & 0.08 & 0.5 \\
Offset $o$ & 0.5 & 15 & 750 & -50 & 15 \\
Maximum firing rate $F$ & 100 & 50 & 200 & 100 & 50
\end{tabular}

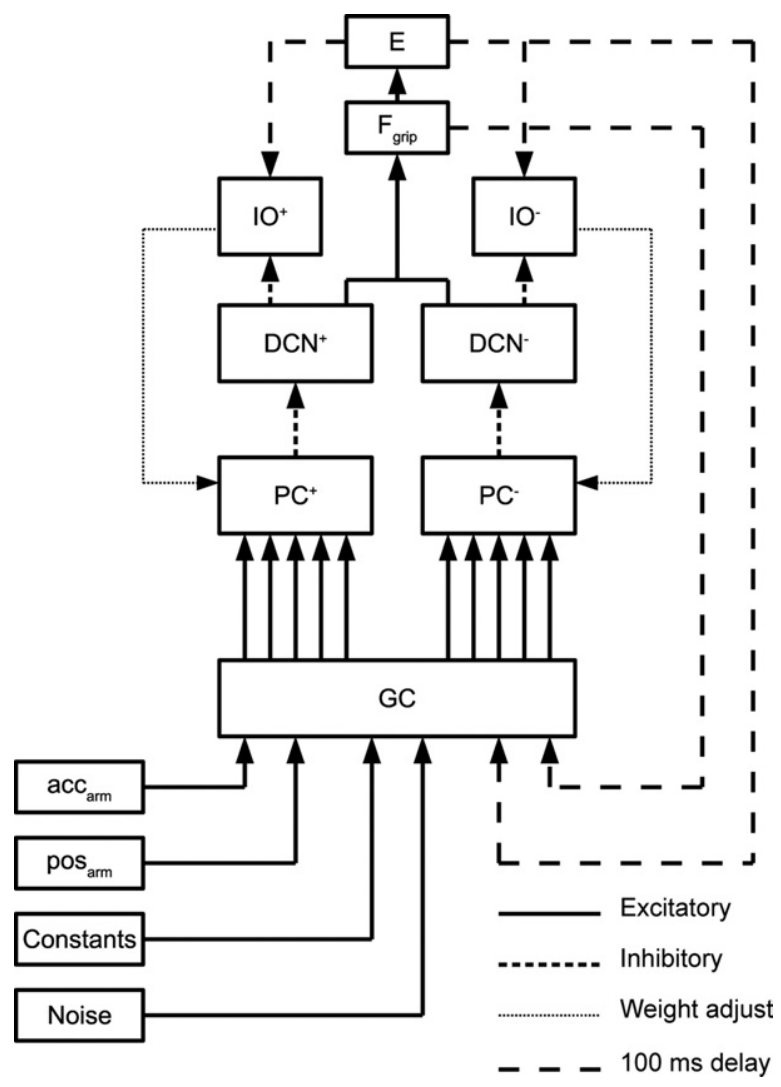

Fig. 2. Overview of the model. For clarity, the interneurons (GO/SC/ $\mathrm{BC})$ are omitted as these cells only serve to normalize the firing rates of the GCs and PCs, respectively. The GC layer acts as an expansion filter, combining MF signals carrying acceleration and position data of

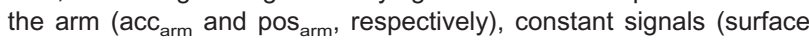
texture and minimum grip force required to prevent slipping when not moving the arm), delayed sensory data on the system's output (grip force $F_{\text {grip }}$ and error $E$ ) and noise. The pre-processed signal is then sent to the PCs. The PC output is processed in the DCN, which generate a change in grip force as output and inhibit the 10 . The sensory apparatus then sends a new perceived state of the system to the GC layer and an error indication to the IO. If the IO neurons fire, the PC's synaptic weights are decreased depending on the local second messenger concentration.

fire units that are hard to excite, so as to be able to take the low number and binary nature of error signals into account.

$$
\text { if } m_{i o}>\Theta
$$

$$
\begin{aligned}
\text { then } m_{i 0} & =m_{i 0}-V_{\text {rebound }} \\
I O & =1 \\
\text { else } I O & =0
\end{aligned}
$$

In the function above, the firing threshold $\Theta$ has the value $[-] 0.0001$ and $V_{\text {rebound }}$ is set at 1.10 is the binary output of the cell. The resulting cell behavior is a baseline firing of approximately $2 \mathrm{~Hz}$, with a maximum firing rate of $10 \mathrm{~Hz}$, in concordance with physiological studies.

The model was modified to fit the current task of grip force control. For the current work, the distribution of the cell population is altered (see Table 2 for the exact num- 
Table 2. Overview of the number of cells used for the model

\begin{tabular}{lr}
\hline Cell type & Cell count \\
\hline Mossy fibers (input cells) & 84 \\
Granule cells & 2100 \\
Golgi cells & 1 \\
Purkinje cells & 40 \\
Stellate or Basket cells & 2 \\
DCN cells & 2 \\
Inferior olivary cells & 2 \\
\hline
\end{tabular}

bers) and the system is not explicitly supplied with a desired goal state as it was by Spoelstra et al. (2000). Rather than specifying the desired grip force, the goal is implicit: use optimal force (derived from arm movement and sensory data) to prevent slipping or crushing of the manipulandum.

Espinoza and Smith (1990) found both cells that increased and cells that decreased their firing rates during lifting of an object. Our model behaves in the same manner by ordering PC groups in an antagonistic fashion. The model is based on the hypothesis that for certain tasks, antagonistic organization of PC groups is vital, with each group receiving a different (opposing) learning signal. This interpretation is also compatible with recent studies which show different cell categories with opposite modulation during trial by trial learning in the vestibulocerebellum (Medina and Lisberger, 2008).

For our experiments we defined 'optimal force' as 'human performance,' since this is robust and reproducible and generally believed to be (almost) optimal (Flanagan and Wing, 1993). The error $E(t)$ can then be computed by subtracting the corresponding grip force value in the dataset $G_{\text {dataset }}$ from the system's grip force output $G_{\text {output }}$ at time step $t$.

$$
E=G_{\text {output }}-G_{\text {dataset }}
$$

Functional organization of PC ensembles is achieved by sending the error $E$ to one of two $I O$ cells depending on its sign. Thus, one such neuron codes for slipping and the other for exerting too much force. If the firing threshold is reached, the $\mathrm{IO}$ cell sends a corrective signal to its corresponding group of PCs. As a result one group of PCs codes for an increase in force, whereas the other codes for a decrease.

The model uses GC expansion recoded data as main input to the PC layer. GCs receive four randomly determined MF inputs each. The MFs are the sole source of quantitative input to the model. Delayed feedback signals are also supplied through the MFs. In Appendix B, the MF signals and distribution are explained in more detail.

The interneurons serve to ensure sparse encoding by keeping activity levels within a certain range. For the GCs this is done by a GO that receives input from the entire GC population and inhibits the very same population in return, ensuring sparse encoding by keeping the summed activity level in the entire population within a certain range. Furthermore, increases in PF activity are compensated for quickly, but not immediately. This way changes can be signaled to PCs in a way reminiscent of the time window hypothesis (D'Angelo and De Zeeuw, 2009). According to this hypothesis, MF signals are translated to well-timed bursts of activity in GCs, the duration of which is regulated by GOs.

Individual PCs receive input from a band of 700 different PFs. Since the PCs have a maximum firing rate and an increase in PF activity could easily cause them to saturate, the SC/BC inhibit the PCs based on the PF activity. This feedforward inhibition causes the PCs to remain in the steeper zones of their sigmoidal activation function even under increased PF activity, thus enhancing their discriminatory capabilities. Furthermore, due to the slightly delayed activation of the SC/BC compared with that of the PCs, the model's feedforward inhibition stresses changes in PF activity and increases temporal precision. This is compatible with results found by Häusser and colleagues (Mittmann et al., 2005).

Feedback signals. In machine learning it is not uncommon that a system receives a quantified value representing its error during learning or even performance. Because of this, we used the value calculated with Equation (4) as a delayed feedback signal. The actual exerted grip force is always supplied to the system as a delayed signal, enabling the system to evaluate its output using the actual value and its deviation from the desired value in this setup, much like a Smith predictor (Miall et al., 1993). (A Smith predictor is a controller for processes involving a time delay. Such a controller uses a forward model to predict the outcome of an action, later comparing this prediction to the actual outcome and making revisions if needed.) Still, these data may not be directly accessible to the cerebellum or a computational model controlling a robot. Therefore, we also investigated two other delayed feedback options: noise (to assess the importance of a delayed feedback signal in general) and values representing fingertip skin deformation (since having error estimation may not be feasible and earlier research indicates that cutaneous feedback is invaluable for correct grip force control (Augurelle et al., 2003; Nowak et al., 2004)). These skin deformations are simulated based on values in the dataset and the output of the system. A description of the model used for this cutaneous input is given in Appendix C.

Learning with a delay. Learning in the system is a result of interplay between long-term potentiation (LTP) and long-term depression (LTD). LTP is the result of PF activity without $\mathrm{IO}$ activity:

$$
\Delta W=\alpha \frac{G C \cdot W}{F_{\max }}
$$

GC represents the GC activity of a population of GCs projecting to a $\mathrm{PC}, W$ the corresponding synaptic weights and $F_{\text {max }}$ is the GC maximum firing rate. The learning speed $\alpha$ is set to $75 \mathrm{e}^{[-] 9}$.

LTD is the result of combined PF and IO activity, the latter of which is caused by sensory feedback with a delay 
of $100 \mathrm{~ms}$ as dictated by biology. The problem of synaptic eligibility that arises as a consequence is tackled with a second messenger $e$, which tags active synapses locally, reaching peak concentration at $100 \mathrm{~ms}$ after synaptic activation. (The problem is accurately tagging the synapses eligible for change. In this case the delay of $100 \mathrm{~ms}$ makes this problematic, since without some form of memory there is no way of detecting which synapses caused undesirable output and need to be corrected.)

$$
\begin{gathered}
\tau_{e 1} \frac{d e_{1}}{d t}=-e_{1}+G C \cdot W \\
\tau_{e} \frac{d e}{d t}=-e+e_{1}
\end{gathered}
$$

The time constants $\tau_{\mathrm{e} 1}$ and $\tau_{\mathrm{e}}$ are both set to 0.1 . Such a system with higher-order dynamics has been suggested before by Schweighofer et al. (1996). This way, no biologically implausible and memory-intensive buffer is needed to represent past activations.

Originally, the second messenger system followed second-order dynamics that resulted in a time span of approximately $250 \mathrm{~ms}$ during which the second messenger concentration e was above $50 \%$ of its maximum caused by a single synaptic activation. In preliminary experiments, this period of time proved to be too long to adequately learn the task of grip force control. Spoelstra et al. (2000) used this form of synaptic eligibility for uni-directional robot arm movements that took $0.6 \mathrm{~s}$ to complete. This task is more constant in nature than grip force control, causing less disruptive spillover of errors. Furthermore, resetting the system between movements can ameliorate most of the problems such a large window of eligibility may cause. The largest time window with which the current task could still be learned to a high degree of precision proved to be approximately $100 \mathrm{~ms}$. Because of this, $e$ is used as the actual second messenger concentration at a certain time $t$, resulting in a period of 100 ms during which the concentration is above $50 \%$ (in every case, the system appears to lag behind the test subject's trace, due to the fact that changes in neural activity are not instantaneous, but occur gradually as a result of the membrane potential and firing rate dynamics defined by Equations (1) and (2); furthermore, test subjects often show changes in grip force more than $10 \mathrm{~ms}$ before the actual change in load force takes place, causing a change in grip force when the model does not yet have sufficient information to do so):

$$
e_{\text {final }}=e^{4}
$$

The final second messenger value can then be used for the LTD learning rule:

$$
\Delta W=\alpha \frac{c e_{\text {final }}}{F_{\text {max }}}
$$

In Equation (9), the learning speed $\alpha$ is set to $[-] 4 \mathrm{e}^{[-] 3}$ and $c$ is a compensatory constant to bring $e_{\text {final }}$ back to the original range of $e$ by approximation.
Changing the firing rate of the $\mathrm{IO}$ (without changing LTP or LTD factors) would cause an imbalance in LTP and LTD, causing one or the other to be more prevalent and making learning impossible in the process. Changing LTP and LTD values along with the inferior olive firing rate, while keeping them balanced, would result in changes in learning speed. Faster learning speeds cause faster, but more inaccurate convergence. Slower learning speeds are more accurate, but converge over a larger time span.

\section{RESULTS}

\section{Training and testing}

Two training sets and two test sets were used. In all three cases (error estimation, fingertip deformation data and noise) the network was trained on 26 of the 35 traces in the dataset. These were presented in random order over 10 iterations, resulting in 260 training sessions in total. After training, the network's state was saved. This was then used as the initial state for testing on each data file of a smaller set of nine trials not present in the training data. To get a reliable estimate of the system's robustness and speed of adaptation, it was tested on trials recorded from subjects that the network had not encountered during training. For test set I these files were all recorded from participant D ( $3 \times$ paper; $3 \times$ Plexiglas; $3 \times$ sandpaper $)$ and for test set II these files were from A $(3 \times$ paper), B $(3 \times$ sandpaper) and $G(3 \times$ Plexiglas).

Test set I consisted of trials that were all more or less average in grip force profiles and grip force levels and may be considered to be the easier test set. Test set II consists of data from three different test subjects, of which one exhibits a highly aberrant grip force level $(G)$ and another has a somewhat unusual grip force profile (A). This test set may also be more difficult due to the fact that the number of such outliers in training set $I I$ is smaller than it is in training set I.

In the following section, an overview of the statistics regarding the performance of each network type is presented. For measure of error the mean squared error (MSE) was used; with the squared error calculated per time step of the simulation and averaged per file, after which it was averaged across all files per iteration. After this overview and its implications, the specifics are illustrated using traces of actual system output plotted against the target (human) output.

\section{Performance specifics}

Performance proved to be mostly dependent on the type of delayed feedback signal. Factors such as surface texture accounted for changes in average grip force levels, but did not cause the system to experience any difficulties in determining necessary changes in grip force. In line with expectations, performance on the second test set is notably worse due to the aberrant data present in this set.

As can be seen in Table 3, only the network using error estimation can learn the training sets to a high degree of precision in 10 iterations. The sensory data and noise models consistently come out of the training cycle with an 
Table 3. Overview of the results. MSE scores of all three systems on the training and test sets

\begin{tabular}{llllr}
\hline $\begin{array}{l}\text { Delayed feedback } \\
\text { signal }\end{array}$ & $\begin{array}{l}\text { Training } \\
\text { set I }\end{array}$ & $\begin{array}{l}\text { Training } \\
\text { set li }\end{array}$ & \multicolumn{1}{l}{$\begin{array}{l}\text { Test } \\
\text { set I }\end{array}$} & \multicolumn{1}{c}{$\begin{array}{l}\text { Test } \\
\text { set li }\end{array}$} \\
\hline Error estimation & $2.5 \pm 0.3$ & $3.1 \pm 0.3$ & $4.5 \pm 1.0$ & $6.3 \pm 1.4$ \\
Skin deformation & $7.4 \pm 0.9$ & $7.3 \pm 0.7$ & $5.6 \pm 1.1$ & $7.1 \pm 1.7$ \\
Noise & $7.1 \pm 1.7$ & $7.1 \pm 1.0$ & $15.2 \pm 8.6$ & $15.1 \pm 5.9$ \\
\hline
\end{tabular}

apparent failure to learn the task well, as indicated by the similarly high MSE scores when compared with the MSE scores of the system with error estimation. However, the difference between noise and sensory data as a delayed feedback signal becomes apparent when the networks' performance on the test sets is compared. As can be seen in Figs. 3, 4 and 5 of typical behavior of the cerebellar model under the three conditions that were investigated, the networks' behavioral properties differ. (In every case, the system appears to lag behind the test subject's trace. This is due to the fact that changes in neural activity are not instantaneous, but occur gradually as a result of the membrane potential and firing rate dynamics defined by Equations (1) and (2). Furthermore, test subjects often show changes in grip force more than $10 \mathrm{~ms}$ before the actual change in load force takes place, causing a change in grip force when the model does not yet have sufficient information to do so.)

The noise model shows high MSE scores with large standard deviations on the test sets, indicating erratic behavior. These networks have been trained on a data set on which they have maximized their performance (albeit with somewhat poor results), but in general fail to extend the learned principles adequately to new data. In addition, the correct baseline grip force is often not found initially. The performance of these systems in general indicates that a delayed signal other than exerted grip force is needed for good grip force control (see Fig. 3).

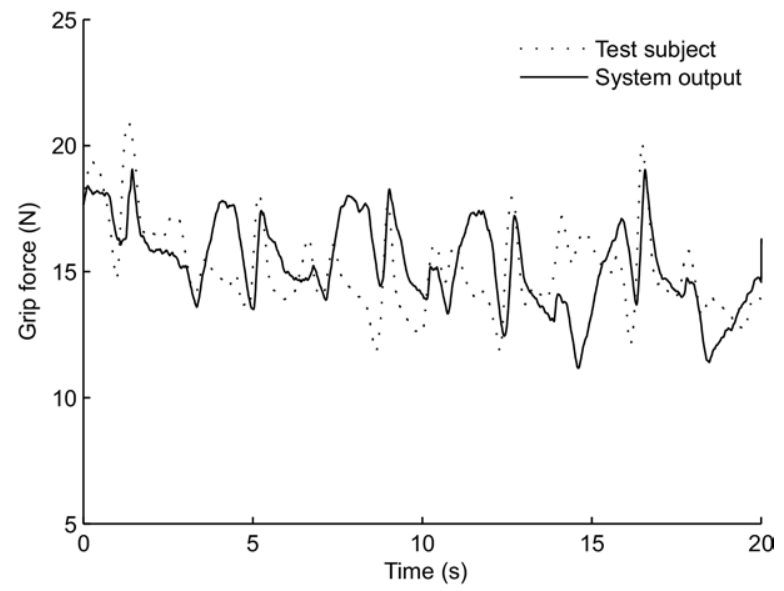

Fig. 3. Trace of a network receiving noise instead of error estimation or fingertip sensory data, being tested on data not present in the training set. The trace shows erratic behavior with missing peaks and force changes where none should occur. Some aspects can still be learned by this model due to the periodic nature of the data, but much of the correct behavior is in response to $\mathrm{IO}$ firing.

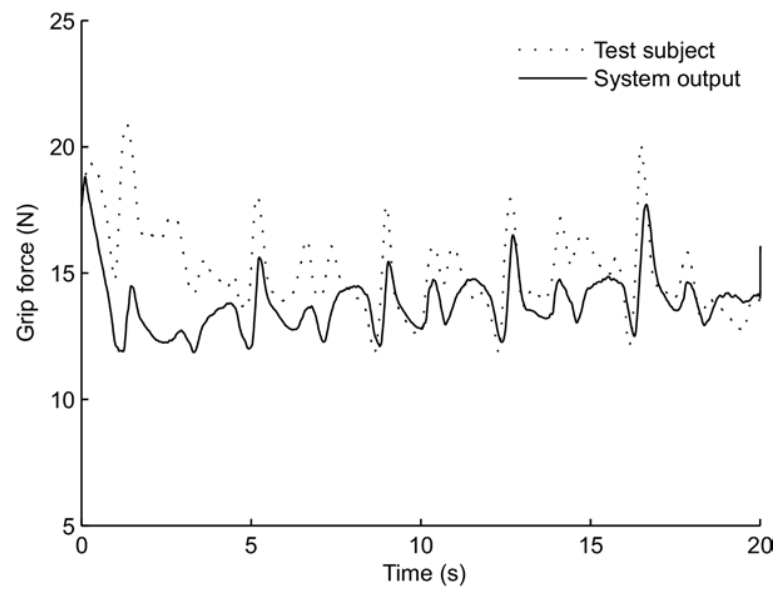

Fig. 4. Trace of a network supplied with delayed information rendered by the fingertip deformation model being tested on data not present in the training set. The grip force characteristics are robust and periodic and adaptation to the new data is fast. However, the system shows lower grip force change amplitudes than networks with other delayed data and the initial grip force level is often not ascertained correctly.

The system using fingertip deformation data has notably lower MSE scores (and much lower variance) than the model supplied with noise on the test sets and shows robust (periodic) grip force control characteristics (see Fig. 4). The signature periodic grip force behavior of these models is an average that would work well in general, because of the astute representation of force changes needed during vertical arm movements as described in the literature (Flanagan and Wing, 1993; Nowak et al., 2002). However, the learned behavior often does not fit traces of individual test subjects well, causing a relatively high MSE score in the training set. In addition, the initial force levels are frequently incorrect, causing the first part of a trial to further increase the average MSE, despite the rapid adaptation of the network to correct the situation. This problem is most severe in test set II, due to the

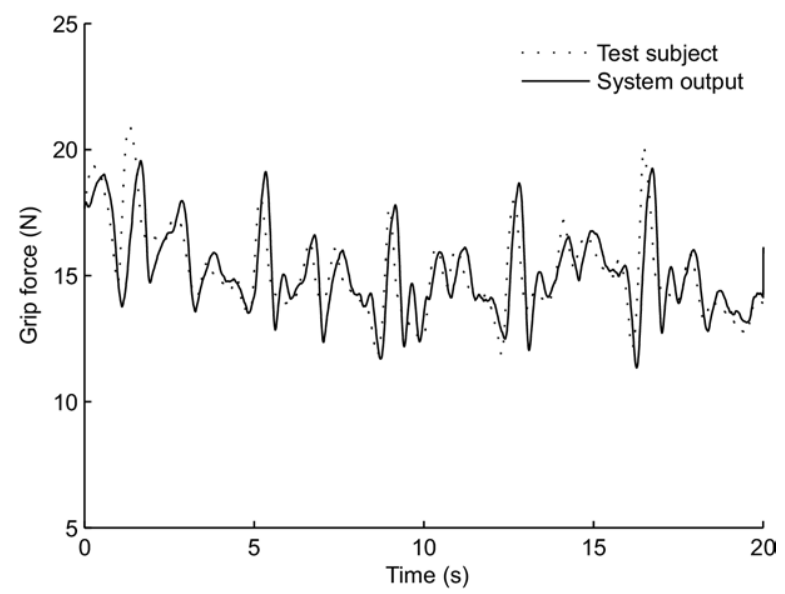

Fig. 5. Trace of a network using error estimation being tested on data not present in the training set. The performance is near perfect, with hardly any sign of learning being necessary to adapt to new data. Some adaptation can be seen to occur in the first 3 seconds. Closer examination reveals that minor grip force changes to fit the test subject's profile often occur in response to $\mathrm{IO}$ firing. 
exceptionally low grip force levels for the Plexiglas surface traces and lack of similar data in the data set. On these data the system consistently uses too much force and fails to adapt quickly enough, resulting in a high MSE score. Since test set I contains new, but no highly aberrant data (contrary to training set I), the averaged behavior that the system exhibits actually causes it to score better on the test set than it does on the training set, with adaptation to the new data occurring quickly.

Networks that use error approximation show increased MSE scores on the test sets when compared with their scores on the training sets, but still perform very well and outperform networks receiving other feedback signals. The numerical representation of the grip force error enables these systems to quickly adapt to specific individual grip force profiles (see Fig. 5). Still, the data for the Plexiglas surface in test set II do cause problems due to the lack of similar traces in training set II. On these data, networks supplied with an estimated error will adapt to the correct baseline grip force level, but tend to increase force too much during movements, resulting in an increased MSE. However, the overall performance of these networks is good. Traces of these systems show extremely fast adaptation to new data and the specific peaks in force associated with the start of an upward movement or the stop of a downward movement that are most critical are almost never missed by this model.

\section{DISCUSSION}

\section{Interpretation of the results}

We tested a model using principles as found in the cerebellum on the task of anticipatory grip force modulation, which is novel for this kind of system. The results of the current work show that this task can be learned to a high degree of precision with a cerebellar model, despite the delayed feedback signal. Even seemingly unnecessary changes in grip force can be anticipated or quickly corrected to. Learning also happens at a fast pace, with a trained system capable of reaching near-optimal performance on new data within seconds.

Learning with a constant delay is possible, but the nature of the delayed signal is important, as can be concluded from the differences in results obtained from the three network types receiving different delayed data. Optimal performance and adaptability are obtained when an accurate estimation of the absolute error in grip force is used, but this may not be biologically plausible. If simulated sensory data on fingertip deformations are used, the model learns a more generalized behavioral pattern and loses the ability to fit every individual trace well. However, the grip force changes rendered as output by this system may still yield good performance on the actual task of holding an object during vertical motion of the arm due to the fact that the temporal pattern that is exhibited is the averaged behavior for such motions as described in the literature (Flanagan and Wing, 1993; Nowak et al., 2002). Use of noise instead of error estimation or simulated sensory data indicates that the delayed input should bear some correlation to the task that is performed in order to learn and maintain accurate control. Earlier research also indicates that without cutaneous feedback grip force control is impaired, resulting in an increased number of dropped objects (Augurelle et al., 2003) or much higher baseline grip force levels (Nowak et al., 2004). Our results show that indeed cutaneous feedback can be used to learn adequate grip force control and that without a feedback signal, the feedforward control fails.

Even though the grip force control characteristics of the model with delayed input representing fingertip deformations look promising, it suffers from the problem of often ascertaining the baseline grip force level needed for a particular trial incorrectly. The model supplied with noise instead of delayed feedback exhibits the same difficulties. This is likely to be caused by the relatively high variance in grip force levels for every individual surface texture used in the dataset. For example: one test subject $(G)$ held the manipulandum with Plexiglas surfaces using grip forces below the average force used for the sandpaper surfaces. The networks receiving error estimation show fewer difficulties in ascertaining such grip force levels, as they can easily use the delayed signal to infer an estimation of the correct baseline grip force. However, if similar data are not present in the training set, ascertaining the correct range for force modulation will still be problematic for these systems.

\section{Neurophysiological questions}

The current model is based on some hypotheses that may be considered controversial. These matters remain to be elucidated by neurophysiological experiments. One is that for learning a corrective CF activation due to slip is used in our model. Dugas and Smith (1992) reported finding no evidence of slip-related complex spikes in PCs in the paravermal anterior lobe that did show simple spike modulation in response or anticipation to load force perturbations during a grip forcerelated task. Still, responses can vary significantly between and even within locations in the cerebellar cortex (Medina and Lisberger, 2008), with cellular activity related to a task often being spread out over the cerebellum. As such, the existence of a slip complex spike signal cannot be excluded.

The link between grip force and the cerebellum has also been a matter of debate. Inactivation of cerebellar nuclei using muscimol in monkeys has yielded conflicting results. Goodkin and Thach (2003) found that muscimol injections in the dentate nucleus can give rise to impaired timing of finger movements, causing delays of up to $50 \mathrm{~ms}$. Monzée et al. (2004) reported no immediate effect (within $20 \mathrm{~min}$ ) of muscimol injections into the dentate nucleus and observed impaired grip force performance on trials where the animal's arm showed tremor and dysmetria and was unrestrained. However, if the forearm was restrained, the grip force behavior was seemingly normal (Monzée et al., 2004). Still, these data are compatible with the possibility that one needs the cerebellum to control multiple motor domains if one controls a particular motor domain (e.g. finger domain) that depends on another domain (e.g. upper arm domain). A lack of reliable data relating to arm movements in areas controlling the fingers could easily give rise to slip. 


\section{Future work}

For future work, it may prove fruitful to improve the model by incorporating results from work on cerebellar interneurons. For instance, recent results show that activity of GOs is coordinated by means of gap junctions (Dugué et al. 2009). These results indicate that input from GC groups can be gated, increasing efficiency of information transfer.

Furthermore, the feasibility of translating sensory data into a motor error indication may be worth investigating. The implementation of a lower-level system that supplies an error signal for self-supervised learning as hypothesized by Spoelstra et al. (2000) would enable the model to function optimally. Due to the regularity of the data during optimal (e.g. human) performance and the clear indication of grip force error that can be rendered using these data and the system's output, it may not be too difficult to train a feedforward function that gives some measure of the actual error that the cerebellar model can use. Having such a signal will also make training a robot easier than when training relies on ascertaining whether an object slips or whether the skin deformation exceeds a certain value for a longer period of time (indicating that too much grip force is being used).

Acknowledgments-We kindly thank the Dutch Organization for Medical Sciences (CIDZ), Life Sciences (CIDZ), Senter (NeuroBsik, CIDZ), Prinses Beatrix Fonds (CIDZ), and the SENSOPAC program of the European Community (CIDZ; JDG; PVDS) for their financial support.

\section{REFERENCES}

Albus JS (1971) A theory of cerebellar function. Mathematical Biosci $10: 1 / 2$.

Albus JS (1975) A new approach to manipulator control: the cerebellar model articulation controller (CMAC). J Dynamic Syst Measure Control 97:220-233.

Augurelle A-S, Smith AM, Lejeune T, Thonnard J-L (2003) Importance of cutaneous feedback in maintaining a secure grip during manipulation of hand-held objects. J Neurophysiol 89:665-671.

Babin-Ratté S, Sirigu A, Gilles M, Wing A (1999) Impaired anticipatory finger grip-force adjustments in a case of cerebellar degeneration. Exp Brain Res 128:81-85.

Boecker H, Lee A, Mühlau M, Ceballos-Baumann A, Ritzl A, Spilker ME, Marquart C, Hermsdörfer J (2005) Force level independent representation of grip force-load force coupling: a PET activation study. Neuroimage 25:243-252.

Cole KJ, Abbs JH (1988) Grip force adjustments evoked by load force perturbations of a grasped object. J Neurophysiol 60:1513-1522.

D'Angelo E, De Zeeuw Cl (2009) Timing and plasticity in the cerebellum: focus on the granular layer. Trends Neurosci 32:30-40.

De Zeeuw CI, Simpson JI, Hoogenraad CC, Galjart N, Koekkoek SKE, Ruigrok TJH (1998) Microcircuitry and function of the inferior olive. Trends Neurosci 21:391-400.

De Zeeuw Cl, Yeo CH (2005) Time and tide in cerebellar memory formation. Curr Opin Neurobiol 15(6):667-674.

Dugas C, Smith AM (1992) Responses of cerebellar Purkinje cells to slip of a hand-held object. J Neurophysiol 67(3):483-495.

Dugué GP, Brunel N, Hakim V, Schwartz E, Chat M, Lévesque M, Courtemanche R, Léna C, Dieudonné S (2009) Electrical coupling mediates tunable low-frequency oscillations and resonance in the cerebellar Golgi cell network. Neuron 61:126-139.
Espinoza E, Smith AM (1990) Purkinje cell simple spike activity during grasping and lifting objects of different textures and weights. J Neurophysiol 64:698-714.

Flanagan JR, Vetter P, Johansson RS, Wolpert DM (2003) Prediction precedes control in motor learning. Curr Biol 13:146-150.

Flanagan JR, Wing AM (1993) Modulation of grip force with load force during point-to-point arm movements. Exp Brain Res 95:131-143.

Goodkin HP, Thach WT (2003) Cerebellar control of constrained and unconstrained movements. I. Nuclear inactivation. J Neurophysiol 89:884-895.

Ito M (1984) The cerebellum and neural control. New York: Raven Press.

Johansson RS, Westling G (1984) Roles of glabrous skin receptors and sensorimotor memory in automatic control of precision grip when lifting rougher or more slippery objects. Exp Brain Res 56: 550-664.

Johansson RS, Westling G (1987) Signals in tactile afferents from the fingers eliciting adaptive motor responses during precision grip. Exp Brain Res 66:141-154.

Llinás R, Volkind RA (1973) The olivo-cerebellar system: functional properties as revealed by harmaline-induced tremor. Exp Brain Res 18:69-87.

Maass W, Natschläger T, Markram H (2002) Real-time computing without stable states: a new framework for neural computation based on perturbations. Neural Comput 14:2531-2560.

Marr D (1969) A theory of cerebellar cortex. J Physiol 202:437-470.

Medina JF, Garcia KS, Nores WL, Taylor NM, Mauk MD (2000) Timing mechanisms in the cerebellum: testing predictions of a large-scale computer simulation. J Neurosci 20(14):5516-5525.

Medina JF, Lisberger SG (2008) Links from complex spikes to local plasticity and motor learning in the cerebellum of awake-behaving monkeys. Nat Neurosci 11(10):1185-1192.

Miall RC, Weir DJ, Wolpert DM, Stein JF (1993) Is the cerebellum a Smith predictor? J Mot Behav 25:203-216.

Mittmann W, Koch U, Häusser M (2005) Feedforward inhibition shapes the spike output of cerebellar Purkinje cells. J Physiol 563(2):369-378.

Monzée J, Drew T, Smith AM (2004) Effects of muscimol inactivation of the cerebellar nuclei on precision grip. J Neurophysiol 91 : $1240-1249$

Nowak DA, Glasauer S, Hermsdörfer J (2004) How predictive is grip force control in the complete absence of somatosensory feedback? Brain 127:182-192.

Nowak DA, Hermsdörfer J, Marquardt C, Fuchs H-H (2002) Grip and load force coupling during discrete vertical arm movements with a grasped object in cerebellar atrophy. Exp Brain Res 145:28-39.

Porrill J, Dean P, Stone JV (2004) Recurrent cerebellar architecture solves the motor-error problem. Proc R Soc Lond B 271:789-796.

Potdar A (2007) Modeling and design of soft fingertips of a dexterous robot hand. Unpublished master's thesis.

Schweighofer N, Arbib MA, Dominey PF (1996) A model of the cerebellum in adaptive-control of saccadic gain. Biol Cybern 75:19-28.

Serrien DJ, Wiesendanger M (1999) Role of the cerebellum in tuning anticipatory and reactive grip force responses. J Cogn Neurosci 11(6):672-681.

Spoelstra J, Schweighofer N, Arbib MA (2000) Cerebellar learning of accurate predictive control for fast-reaching movements. Biol Cybern 82:321-333.

Van Der Giessen RS, Koekkoek SK, van Dorp S, De Gruijl JR, Cupido A, Khosrovani S, Dortland B, Wellershaus K, Degen J, Deuchars J, Fuchs EC, Monyer H, Willecke K, De Jeu MT, De Zeeuw Cl (2008) Role of olivary electrical coupling in cerebellar motor learning. Neuron 58(4):599-612.

Wolpert DM, Miall RC, Kawato M (1998) Internal models in the cerebellum. Trends Cogn Sci 9:338-347.

Yamazaki T, Tanaka S (2007) The cerebellum as a liquid state machine. Neural Netw 20:290-297.

Yeo $\mathrm{CH}$, Hesslow G (1998) Cerebellum and conditioned reflexes. Trends Cogn Sci 9:322-330. 
APPENDIX A: HUMAN GRIP FORCE BEHAVIOR DATASET

The following data describe the dataset used to train and test the cerebellar model.

\begin{tabular}{|c|c|c|c|}
\hline Subject & Sandpaper trials & Plexiglas trials & Paper trials \\
\hline A & 0 & 0 & 3 \\
\hline B & 3 & 0 & 0 \\
\hline C & 0 & 1 & 0 \\
\hline D & 3 & 3 & 3 \\
\hline$E$ & 3 & 0 & 0 \\
\hline $\mathrm{F}$ & 1 & 1 & 1 \\
\hline G & 0 & 3 & 0 \\
\hline $\mathrm{H}$ & 3 & 3 & 3 \\
\hline 1 & 0 & 0 & 1 \\
\hline Total: & 13 & 11 & 11 \\
\hline
\end{tabular}

B: Averages of grip force per surface texture.

\section{APPENDIX B: SIMULATED MF INPUT}

A total of 84 MFs was used for the model. The MF input supplied to the model consists of a predetermined part (60 inputs, derived from the dataset) and a dynamic part (24 inputs, based on system output). The values based on system output are considered to be of sensory origin and arrive with a $100 \mathrm{~ms}$ delay. Most signals were distributed over several (gaussian) receptive fields responding to values in a certain range, generating a value in the range of $[0 \ldots 1]$ as output.

\section{Predetermined MF input}

$3 D$ accelerations. The 3D acceleration signals were spread over receptive fields, using five fields for the $x$ and $z$ (horizontal plane) axes and nine fields for the $y$ axis. These receptive fields span the total range of values for accelerations found in the dataset. Every receptive field was included twice as a MF input, resulting in 38 acceleration inputs.

\section{Arm position}

The torque signal was used as an indication of arm position, since the torque forces closely resembled the movement due to rotational friction caused by movement of the shoulder during vertical motions of the extended arm. The raw data were normalized and spread over three receptive fields coding for low, middle and high position. A total of nine arm position inputs were used (three low, three middle, three high).

\section{Minimum grip force}

This value was obtained by letting the test subject lessen the grip force on the manipulandum while holding it and using the telltale increase in grip force in the raw trace as a marker for the beginning of slip. This constant is used to indicate when the object would slip based on surface friction only. The signal is spread over four receptive fields covering the entire range of minimum grip force values in the dataset. A total of eight minimum grip force inputs was used (two for every receptive field).

\section{Noise}

A total of three inputs supplying white noise was used.

\section{Surface texture}

A total of two inputs coding for the surface texture was used, with outputs 0 for sandpaper, 0.5 for Plexiglas and 1 for paper.

\section{Dynamic MF input}

Exerted grip force. The actual exerted grip force is spread over eight receptive fields, spanning a little more than the entire range of grip force values in the dataset. The output of every receptive field is included twice, for a total of 16 grip force inputs.

\section{PC activity intensity}

This input codes for the activity in PC groups, with one value per group. The value in the range [0 . . 1] is determined by the theoretical maximum and minimum output of these groups, divided by the maximum. Every input is included twice, for a total of four inputs. 


\section{Feedback signal}

Information on error estimation, skin deformation or noise carried by a total of four inputs. Error estimation: two inputs for overgripping and two inputs for slipping. Skin deformation: two inputs for fingertip contact area and two inputs for fingertip deformation in the plane of the contact area.

\section{APPENDIX C: FINGERTIP SKIN DEFORMATION MODEL}

When a digit is put to a surface and force is applied, the contact area expands until a maximum is reached. Elastic materials such as skin tissue tend to show an instantaneous elastic response followed by relaxation behavior. In the case of a contact area, this means that there is an instantaneous increase of the area due to force, after which the material will settle and relax, causing a slow increase of the surface area dependent on time. To simulate the force dependency of the total contact area and the visco-elastic properties of the finger's tissue, the following iterative functions are used:

$$
\begin{gathered}
A_{\infty}(t)=\frac{1}{1+e^{F(t) / 8}} \\
A(t)=A(t-1)+\frac{A_{\infty}(t)-A_{\infty}(t-1)}{i}+\frac{A_{\infty}(t)-A(t-1)}{V}
\end{gathered}
$$

with $A(t)$ as the contact area at time $t$ and $F(t)$ the actual force in newtons with which the finger is pressed onto the surface at time $t$. The constants $i=1.4286$ and $v=40$ define the amount of instantaneous elastic response and the time-dependent gradual increase or decrease rate of the surface area due to the viscous-like properties, respectively. The resulting behavior is an immediate elastic response that accounts for $70 \%$ of the change due to force changes, followed by relaxation behavior that settles in $2 \mathrm{~s}$. This approximates the properties of silicone rubber such as may be used for the construction of the fingertips of a dexterous robot hand (Potdar, 2007). In order to perpendicularly lift the sensor, the surface friction must overcome the gravitational force. This surface friction is the result of the fingertip's and the surface area's friction coefficients as well as the size of the contact area. If the minimum force to prevent slipping $F_{0}$ is used in Equation (10), the initial contact area $A(0)$ is found. $A(t)$ is also used as a direct measure of how compressed the fingertip is. The more compressed the fingertip is, the harder it is to deform:

$$
\begin{gathered}
\delta_{\infty}(t)=F_{a}(t)-F_{a}(t)\left(1-A^{2}(t)\right) \\
\delta(t)=\delta(t-1)+\frac{\delta_{\infty}(t)-\delta_{\infty}(t-1)}{i}+\frac{\delta_{\infty}(t)-\delta(t-1)}{V}
\end{gathered}
$$

The deformation $\delta(t)$ along an axis perpendicular to the axis along which grip force is exerted is determined by the surface area $A(t)$ and the total acceleration force $F a(t)$ in that particular direction. $F_{\mathrm{a}}$ is normalized to values between -1 and 1 . The deformations in both possible directions $(x$ and $y$ ) are assumed to be independent. Note that in the vertical direction $y, F a(0)<0$ is due to gravity and that the fingertip is initially deformed as a consequence. The final information vector that is sent to the GC layer via the MFs is $<A(t), \delta x(t), \delta y(t)>$.

(Accepted 19 February 2009) 